\title{
Band Positions of Anatase (001) and (101) Surfaces in Contact with Water from Density Functional Theory
}

Julian Geiger, Michiel Sprik, Matthias May

Submitted date: 07/05/2020 - Posted date: 08/05/2020

Licence: CC BY-NC-ND 4.0

Citation information: Geiger, Julian; Sprik, Michiel; May, Matthias (2020): Band Positions of Anatase (001) and (101) Surfaces in Contact with Water from Density Functional Theory. ChemRxiv. Preprint. https://doi.org/10.26434/chemrxiv.12263837.v1

Titanium dioxide in the anatase configuration plays an increasingly important role for photo(electro)catalytic applications due to its superior electronic properties when compared to rutile. In aqueous environments, the surface chemistry and energetic band positions upon contact with water determine charge-transfer processes over solid--solid or solid--electrolyte interfaces. Here, we study the interaction of anatase (001) and (101) surfaces with water and the resulting energetic alignment by means of hybrid density functional theory. While the alignment of band positions favours charge-transfer processes between the two facets for the pristine surfaces, we find the magnitude of this underlying driving force to crucially depend on water coverage and degree of dissociation. It can be largely alleviated for intermediate water coverages. Surface states and their passivation by dissociatively adsorbed water play an important role here. Our results suggest that anatase band positions can be controlled over a range of almost one $\mathrm{eV}$ via its surface chemistry.

File list (2)

Band_positions_anatase.pdf (4.85 MiB) view on ChemRxiv - download file 


\title{
Band Positions of Anatase (001) and (101) Surfaces in Contact with Water from Density Functional Theory
}

\author{
Julian Geiger, ${ }^{1,2}$ Michiel Sprik, ${ }^{1,}$ a) and Matthias M. May ${ }^{1,3}$ \\ 1)Department of Chemistry, University of Cambridge, United Kingdom \\ 2) Friedrich-Alexander University Erlangen-Nürnberg, Germany ${ }^{\text {b) }}$ \\ ${ }^{3)}$ Institute for Solar Fuels, Helmholtz-Zentrum Berlin für Materialien und Energie GmbH, \\ Germany $^{\mathrm{c})}$
}

Titanium dioxide in the anatase configuration plays an increasingly important role for photo(electro)catalytic applications due to its superior electronic properties when compared to rutile. In aqueous environments, the surface chemistry and energetic band positions upon contact with water determine charge-transfer processes over solid-solid or solid-electrolyte interfaces. Here, we study the interaction of anatase (001) and (101) surfaces with water and the resulting energetic alignment by means of hybrid density functional theory. While the alignment of band positions favours charge-transfer processes between the two facets for the pristine surfaces, we find the magnitude of this underlying driving force to crucially depend on water coverage and degree of dissociation. It can be largely alleviated for intermediate water coverages. Surface states and their passivation by dissociatively adsorbed water play an important role here. Our results suggest that anatase band positions can be controlled over a range of almost one $\mathrm{eV}$ via its surface chemistry.

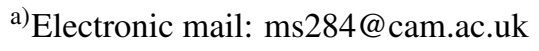

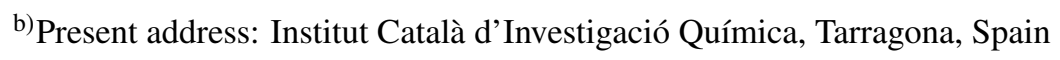

c) Present address: Institute of Theoretical Chemistry, Ulm University, Germany
} 


\section{INTRODUCTION}

Titania $\left(\mathrm{TiO}_{2}\right)$ is one of the most prominent materials in (electro)catalysis, notably in the function as a photocatalyst or protection layer for solar water splitting. ${ }^{1,2}$ Here, the application case crucially depends on the energetic alignment of the bands with respect to the electrolyte or the underlying photoabsorber. Furthermore, titanium dioxide is the model system par excellence for a wide-gap metal-oxide to study the electronic properties of a semiconductor in contact with water, both experimentally ${ }^{3-5}$ and theoretically, ${ }^{4,6-16}$ hereby also playing an important role in method development and validation.

Anatase is, though metastable, considered the more interesting polymorph of $\mathrm{TiO}_{2}$ for photocatalysis as it exhibits superior electronic properties, manifested in longer charge-carrier lifetimes when compared to rutile. ${ }^{17}$ In a density functional theory (DFT)-based molecular dynamics study, evidence was found for a trapping mechanism of excess electrons on the anatase (101) surface, whereas the (001) surface acts as hole acceptor. ${ }^{12}$ Experimentally, photoelectron spectroscopy at differently treated (101) and (001) surfaces of single-crystals evidenced a band offset favouring electron migration to the (101) facets, whose magnitude depends on prior sample treatment. ${ }^{18,19}$ Such a driving force could help in improving the catalytic activity of $\mathrm{TiO}_{2}$ nanoparticles or improve the charge-transport over hetero-interfaces for $\mathrm{TiO}_{2}$-based protection layers. A key question, to be addressed in this study, is to what extent the contact with water modifies this surface electronic structure behaviour.

In this work we have considered the (101) and (001) surfaces of anatase, as illustrated in Fig. 1. The (101) surface is characterised by a ridge-like structure with every second titanium atom being coordinatively saturated and buried in the trenches of the surface steps. The other half entail a five-fold coordination, exposing one free coordination site that can function as anchoring point for adsorbate molecules. In contrast, all titanium and half of the oxygen atoms constituting the (001) surface are coordinatively unsaturated, with the uppermost oxygen atoms bearing large bond angles of around $150^{\circ}$. The high degree of unsaturation and the strained bonding situation lead to the well-known increased reactivity of this surface. It can undergo reconstruction under ultra-high vacuum conditions, ${ }^{20}$ yet also the unreconstructed surface has been observed. ${ }^{19}$ As adsorption of water prevents reconstruction in an aqueous environment, ${ }^{21,22}$ the slab models employed in this work correspond to the unreconstructed surface. 


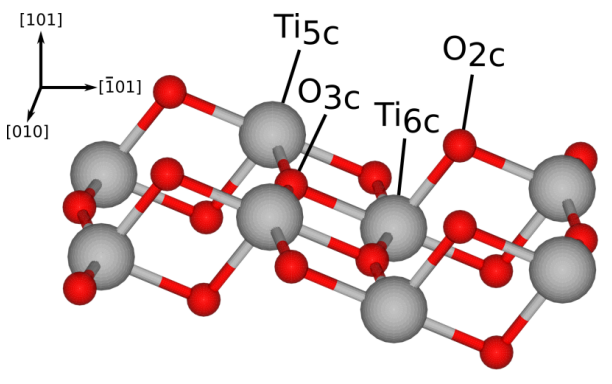

(a) (101)

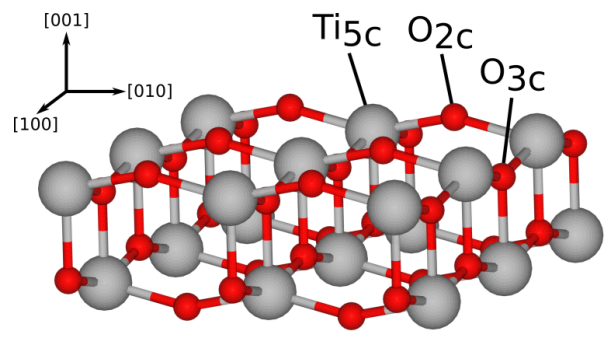

(b) (001)

FIG. 1. Ball-and-stick models of the atomic upper layers for the considered anatase surfaces.

Experimental and theoretical work on the initial water layer in contact with anatase surfaces found a mixture of dissociative and molecular adsorption, with temperatures beyond $120 \mathrm{~K}$ initiating dissociation on (101) surfaces. ${ }^{5}$ Furthermore, intermediate water coverages are believed to lower the dissociation barrier. ${ }^{23}$ Slow adsorption at low temperatures, can, however, result in the formation of an ordered, full coverage of molecular water. ${ }^{5}$ A detailed insight into the interplay between surface chemistry and surface electronic states is required to understand the interfacial energetic alignment between solid and electrolyte, as well as different facets of anatase (nano)crystals.

Here, we present electronic structure calculations of titanium dioxide surfaces in the anatase structure, investigating the energetic band alignment with and without (fractional) water coverage. We find that facet-driven charge-carrier separation is favoured in a static picture by band-offset. However, band positions can be modified significantly through the adsorption of water. The magnitude of the surface chemistry-induced shifts of the band levels depends on coverage and degree of dissociation and can be in the order of up to one $\mathrm{eV}$.

\section{METHODS}

We performed DFT electronic ground state calculations with the CP2K code, ${ }^{24}$ employing the Gaussian-And-Plane-Wave scheme with GTH pseudopotentials. The Gaussian basis sets were of double- $\zeta$ quality $^{25}$ and an energetic cutoff of $600 \mathrm{Ry}$ was used. Initial optimisations of our slabs were carried out within the generalised gradient approach (GGA), using the PBE functional. ${ }^{26,27}$ The resulting structures were then subjected to further relaxation with the HSE06 hybrid functional, using the auxiliary density matrix method as implemented in $\mathrm{CP} 2 \mathrm{~K} .{ }^{28,29}$ In the subsequent hybrid functional optimisations, all atoms were allowed to relax further, while keeping the cell 
sizes fixed at the PBE-calculated values. Total energies were evaluated at the $\Gamma$-point. For the (001) surface, the in-plane dimensions of our model systems comprised four unit cells in $x$ - and $y$-direction, extending $15.08 \AA$ in each direction. The (101) surface was modeled by supercells composed of one unit cell in $x$ - and three in $y$-direction, corresponding to dimensions of $10.26 \AA$ and $11.31 \AA$, respectively. The slabs were separated by $20 \AA$ of vacuum along the $z$-direction. No constraints on atomic positions were applied and the full slabs were allowed to relax. For the geometry optimisations, we first optimised the crystal-structures in vacuum, with, in the case of the water-covered surfaces, a subsequent further relaxation after addition of 0.25 (in the case of the (001) surface), 0.5 , or 1.0 monolayers (ML) of water. Water molecules were initially positioned on the surfaces in a way to facilitate coordination of the oxygen atoms to the under-coordinated titanium atoms and to allow for hydrogen bonding with the surface oxygen atoms. ${ }^{30,31}$ Based on full ML coverage, removal of certain water molecules resulting in high-symmetry over-structures provided starting points for optimisations with sub-ML coverages. Additionally, we varied the number of Ti-layers along the $z$-direction between 8 and 20 to investigate band gap convergence and to confirm the absence of any intrinsic dipole of the surfaces. For these considerations we applied the PBE functional and, in the case of the (001) surface, a smaller 3 by 3 supercell. Presented surface structures, band gap values, adsorption energies, heats of formation and partial densities of states (PDOS) were obtained using slabs with 20 Ti-layers in the $z$-direction and the HSE06 hybrid functional. These structures were chosen to be in a well-converged regime for band positions and energy gaps, as confirmed beforehand.

\section{RESULTS AND DISCUSSION}

The main structures resulting from our geometry optimisations for the different water coverages are shown in Figs. 2 and 3 and Supplementary Figs. 1 and 2. Irrespective of the coverage, we obtain only molecular adsorption on the (101) surface. This is consistent with previous reports, ${ }^{31}$ in which molecular adsorption was identified as energetically favourable over dissociative adsorption. In the resulting structures, the water molecules are coordinated to the surface titanium atoms and hydrogen bonds to the surface oxygen atoms are formed.

For full ML coverage on the (001) surface, on the other hand, we observe partial dissociation,

as shown in Fig. 3a. The resulting structure is similar to a previously reported result, ${ }^{7}$ in which adsorption of one ML of water on the (001) surface led to the dissociation of one quarter of the wa- 


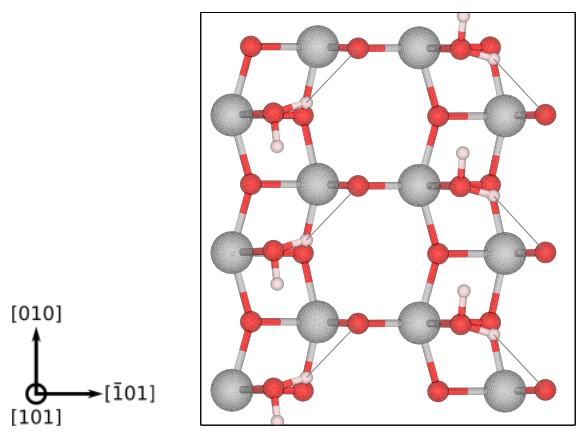

(a) $1 \mathrm{ML}$

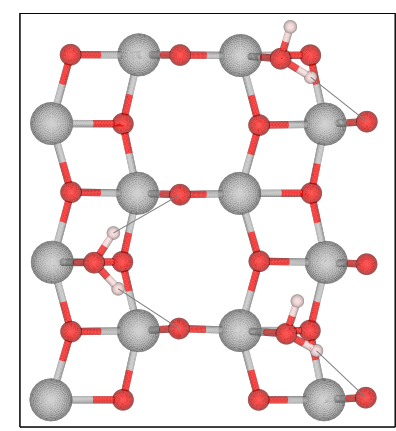

(b) $1 / 2 \mathrm{ML}$

FIG. 2. Top view of water adsorbed on the (101) surface, with one (a) and half a monolayer (b) coverage. Hydrogen bonds are represented as thin grey lines.

ter molecules. The remaining molecularly adsorbed water forms complex networks of hydrogen bonds, also including surface oxygen atoms. This structure was obtained after application of the HSE06 hybrid functional, whereas initial optimisation with PBE resulted in an ordered, fully nondissociated, adsorbed layer of water. For half ML coverage, we observed mixed molecular and dissociative adsorption, Fig. 3b, while quarter ML coverage led to fully dissociative adsorption, Fig. 3c. In both of these cases, the resulting total number of surface hydroxyl groups is the same. Dissociative water adsorption presents an energetically favourable process, as it alleviates some of the strain caused by the large bond angles around the oxygen atoms present in the pristine surface. The final structures are further stabilised by hydrogen bonds between the surface hydroxyl groups, if present, also including the molecularly adsorbed water. Interestingly, the geometric motif around the pairs of surface hydroxyl groups shows a close similarity to the common 1 x 4 reconstruction of the pristine (001) surface, as previously reported. ${ }^{7}$

Mixed dissociative adsorption of water on the (001) surface allows for the formation of various geometrically distinct final structures, differing in the relative positioning of the resulting surface hydroxyl groups. While we have not realized an extensive search over these geometrically distinct structures, we have considered various representative examples for each water coverage. We found their differences in relative stability, water adsorption energy and electronic structure to be negligible. Experimentally, we expect them, in principle, to be distinguishable by surface-sensitive methods such as low-energy electron diffraction. However, for real surfaces at finite temperatures, different superstructures might co-exist.

To assess stability and likelihood of actual occurrence, we calculated heat of formation energies, 


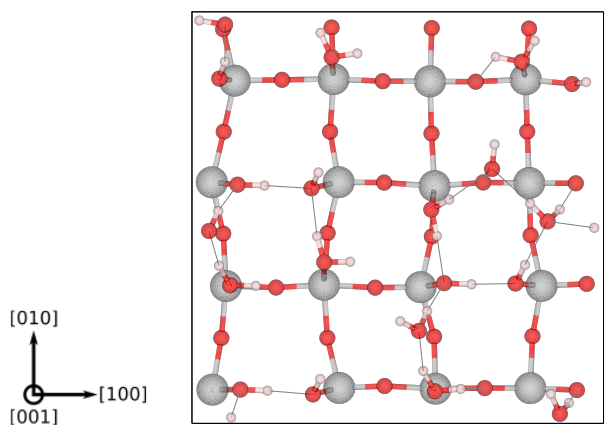

(a) $1 \mathrm{ML}$

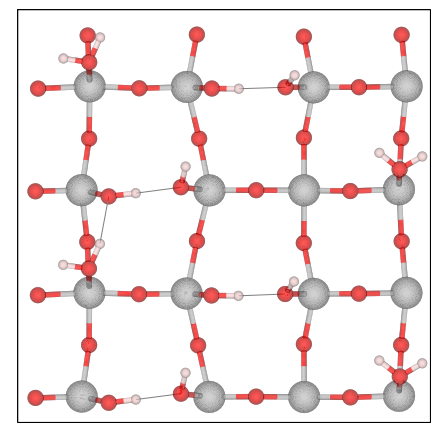

(b) $1 / 2 \mathrm{ML}$

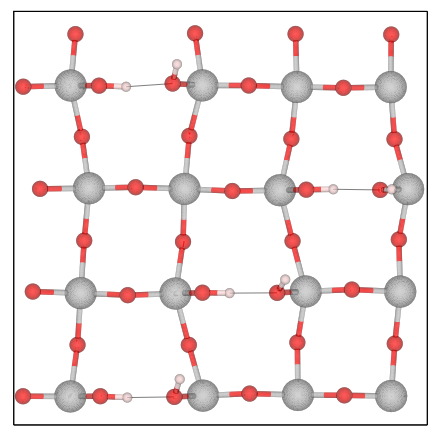

(c) $1 / 4 \mathrm{ML}$

FIG. 3. Top view of mixed (a,b) and fully dissociative (c) water adsorption on the (001) surface from different water coverages.

$\Delta H_{f}(\theta)$, and adsorption energies, $E_{a d s}$, as a function of the coverage, $\theta$. The resulting values per $(1 \times 1)$ surface unit cell are listed in Table I for different coverages and different degrees of dissociation. The heat of formation energy as a measure for relative stability is obtained from the total energies, $E^{\text {tot }}$, neglecting the $p V$ term and vibrational energies, ${ }^{32}$ as follows:

$$
\Delta H_{f}(\theta)=E^{t o t}(\theta)-E^{t o t}(0)-\theta\left[E^{t o t}(1)-E^{t o t}(0)\right]
$$

Adsorption energies per water molecule were calculated as:

$$
E_{a d s} \cdot n_{H_{2} O}=E^{t o t}(\theta)-\left[E^{t o t}(0)+n_{H_{2} O} \cdot\left(E_{H_{2} O, g}^{t o t}\right)\right]
$$

with the total energy of a single gas-phase water molecule, $E_{H_{2} O, g}^{t o t}$, and the number of water molecules, $n_{H_{2}} \mathrm{O}$. In general, our values of the adsorption energies show a similar trend as reported in the literature. ${ }^{22,31}$ For the (001) surface, the most favourable structure at a quarter of a ML is a fully dissociated adsorption of water molecules, while for $0.5 \mathrm{ML}$, half of the water molecules stay intact, leading to a comparable heat of formation.

Densities of states for the (001) surface with different water coverages are shown in Fig. 4. It should be noted that the seemingly large magnitude of the band gaps is due to the fact that the lowest unoccupied states are too small to be visible in this representation. However, they lie in fact about $3.2 \mathrm{eV}$ to $3.6 \mathrm{eV}$ above the valence band maximum, as expected. Analysis of the density of states shows that the pristine (001) surface exhibits an occupied Ti-related surface state in the band gap region close to the valence band maximum, which reduces the effective band gap. It can be seen in Fig. 4a) and is clearly visible in the local density of states of the very top Ti-O 
TABLE I. Heat of formation energies (per surface unit cell) and adsorption energies (per water molecule). Different degrees of dissociation are labelled as fd: fully dissociated, pd: partially dissociated, and ud: undissociated.

(001) surface

\begin{tabular}{l|l|l}
\hline \hline Water coverage / ML & $E_{a d s} / \mathrm{eV}$ & $\Delta H_{f} / \mathrm{eV}$ \\
\hline $0.25 \mathrm{fd}$ & -2.34 & -0.35 \\
$0.25 \mathrm{pd}$ & -1.92 & -0.25 \\
$0.5 \mathrm{pd}$ & -1.43 & -0.25 \\
$1.0 \mathrm{pd}$ & -0.94 & 0.00 \\
\hline
\end{tabular}

(101) surface

\begin{tabular}{l|l|l}
\hline \hline 0.5 & -0.77 & -0.04 \\
1.0 & -0.73 & 0.00 \\
\hline
\end{tabular}

layer (Supplementary Fig. 3). This occupied surface state leads to a charge redistribution between surface and bulk, hereby contributing to the shift of band positions (see below). However, dissociative adsorption of water causes the disappearance or a 'passivation' of this state, as can be seen in Fig. 4b,c. For the (101) surface, this state is absent, resulting in virtually unchanged Ti-related valence band features and a constant band gap.

This is similar to the experimental photoelectron spectroscopy results of Kashiwaya et al. ${ }^{18}$ : Their study on differently prepared single-crystals of anatase with X-ray and ultraviolet photoemission in vacuum find occupied surface states slightly above the bulk valence band maximum for the (001) surface that can be - apart from a shallow valence band surface state - largely suppressed by dedicated sputter-annealing routines followed by reoxidation. Their (001) surface without reoxidation still comprises a deep band gap state very close to the middle of the band gap. Though they consider this type of surface "stoichiometric", our results show that the occupied surface state for the pristine (001) surface is very close to the highest occupied bulk states. Consequently, the signatures of their "oxidised" surface are closer to the pristine, unreconstructed surface. In the case of the (101) surface, the "stoichiometric" samples would indeed correspond to the pristine configuration, which means that the annealing procedure creates oxygen vacancies that have to be cured by dedicated oxidation. 

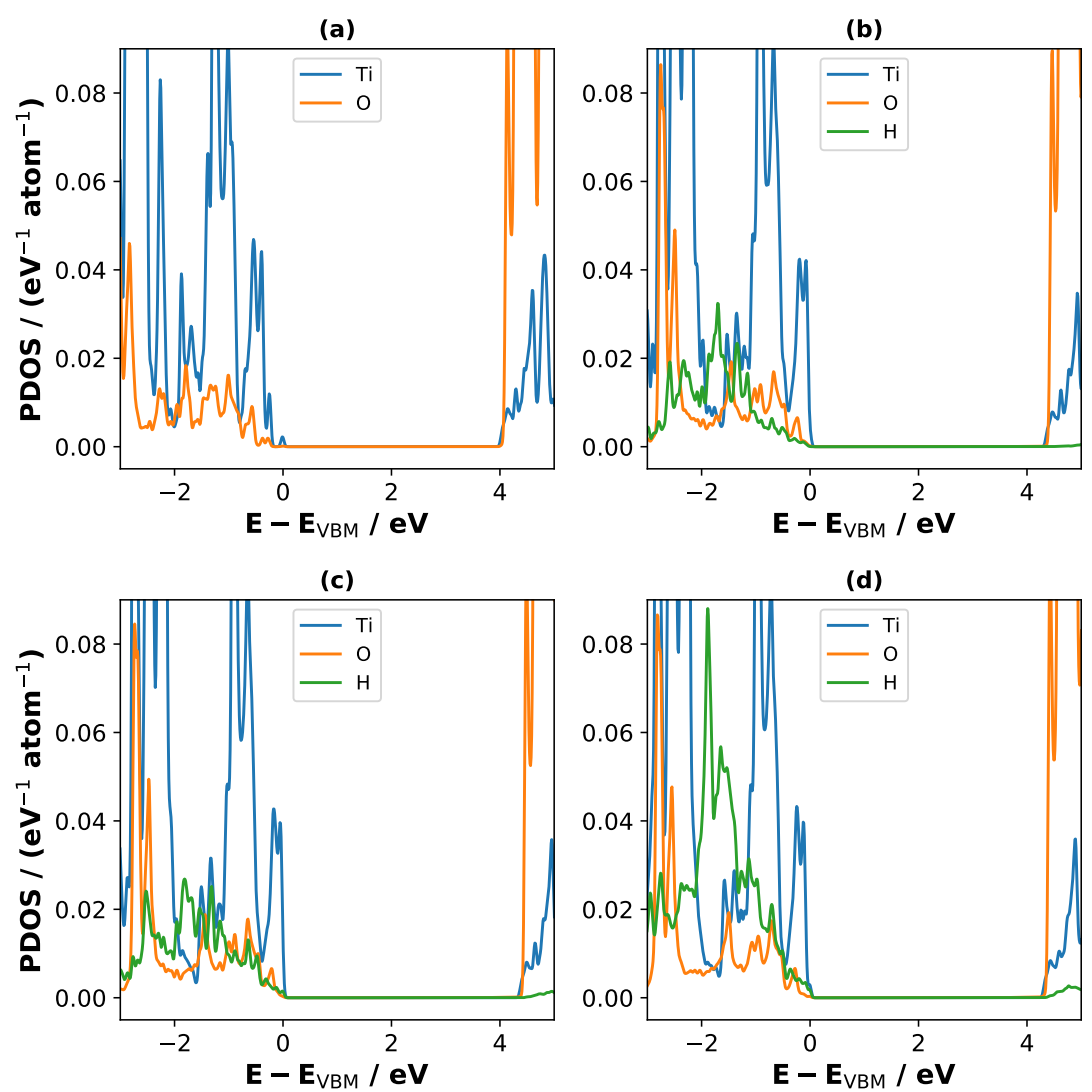

FIG. 4. Partial density of states (PDOS) for the lowest-energy (001) surfaces. (a) without water, (b) with a quarter ML of fully dissociated water, (c) with half a ML of partially dissociated, and (d) with a full ML of partially dissociated water. Energies are referred to the valence band maximum, $E_{\mathrm{VBM}}$.

The band gaps of the different structures are presented in Fig. 5. These were obtained by taking the difference between the conduction band minimum (CBM) and the valence band maximum (VBM) for each system. As expected, the gaps for the (101) surface are with $3.64 \mathrm{eV}$ largest and virtually unaffected by water in the vicinity of the surface. This value represents the well-known, slight overestimation of HSE06 in comparison to the experimental band gap. ${ }^{33}$ The picture for the (001) surface is, however, a completely different one. The pristine, unreconstructed surface shows a reduced gap of $3.24 \mathrm{eV}$, which can be attributed to the previously discussed surface state. The band gap continuously increases with the number of dissociatively adsorbed water molecules. While partial dissociation of a quarter ML yields a value of $3.32 \mathrm{eV}$, about $80 \mathrm{meV}$ above the gap of the pristine surface, fully dissociative adsorption causes a strong increase to $3.57 \mathrm{eV}$, over $330 \mathrm{meV}$ above the pristine surface. In the case of partial dissociation of half a ML of water, the 
total concentration of surface hydroxyl group is the same. Thus, the band gap for this system is with $3.59 \mathrm{eV}$ nearly identical. These values almost reach the band gap magnitude of the (101) surface.

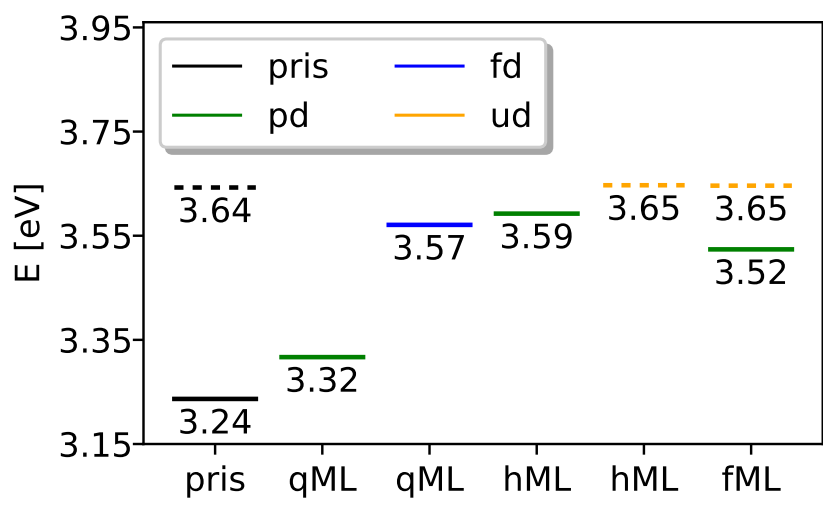

FIG. 5. Band gaps for (001) and (101) surfaces of anatase, represented as solid and dashed lines, respectively. Different degrees of water dissociation are indicated by different colouring.

More relevant for charge-transfer processes between crystal facets or to electrolytes are, however, the relative and absolute energetic positions of valence and conduction bands. To obtain the absolute energetic positions of the VBM and CBM, we subtracted the Hartree potential in the vacuum region, projected onto the $z$-axis, from the HOMO and LUMO energies of the DFT calculations. This referencing has initially no physical meaning in a 3D periodic calculation. However, as the vacuum level of the Hartree potential is dependent on the crystal-to-vacuum ratio, it converges to a specific value with the number of layers (not shown here). Thus, the dependency on the number of layers in the crystal - keeping the amount of vacuum fixed - cancels and the obtained values quickly converge with slab thickness.

Figure 6 shows the resulting band positions with respect to the Hartree potential at vacuum for each system. For the pristine surfaces, the CBM of the (101) surface lies $600 \mathrm{meV}$ lower than for the (001) surface. With a value of $1 \mathrm{eV}$, this difference is even more pronounced for the VBM. In a nanocrystal, where both surfaces can be present on different facets, the band position difference of the two facets represents an intrinsic driving force for electron-hole separation after photoexcitation. ${ }^{12}$ The hole should be more stable in the (001) system, whereas the electrons will be trapped in the lower energy levels of the (101) conduction band. However, we find that 
the electronic structures of the surfaces exposed by such a nanocrystal can be influenced considerably by the presence of water. While dissociative adsorption on the (001) surface mainly causes a decrease of the VBM level, molecular adsorption on both surfaces leads to an almost linear increase of both band positions. This is especially evident in the case of the (101) surface, where the band levels for the fully covered surface lie about $1.3 \mathrm{eV}$ higher than for the pristine surface. The resulting position of the $\mathrm{CBM}$ at $-3.75 \mathrm{eV}$, as compared to a value of $-4.02 \mathrm{eV}$ for the fully covered (001) surface, suggests a higher stability of electrons in the (001) surface. This stands in contrast to the previously mentioned trapping of electrons at the (101) facets. Similarly, coverage of half a ML of water causes the band positions of both surfaces to approach values of about $-7.9 \mathrm{eV}(\mathrm{VBM})$ and $-4.3 \mathrm{eV}(\mathrm{CBM})$, again reducing the driving force for electron-hole separation. Comparison of different coverages and degrees of dissociation for both surfaces, however, shows that the intrinsic band level differences between the two surfaces can also be further increased. For instance, full ML coverage on the (001) surface leads to an overall increase of band positions and therefore enhances the band level differences to the pristine (101) surface. This should in turn further improve the efficiency of electron-hole separation after photoexcitation.

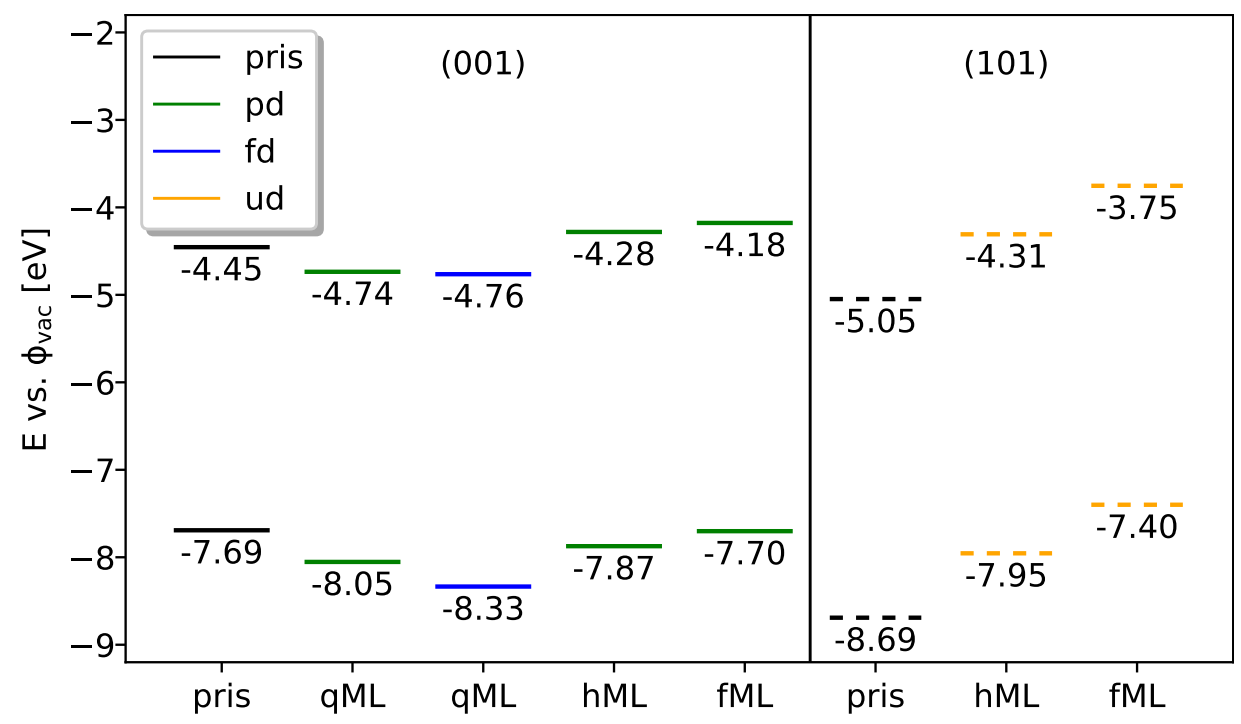

FIG. 6. Band positions for (001) and (101) surfaces of anatase. VBM and CBM levels are represented as solid and dashed lines, respectively.

Our findings are in good agreement with the results of Kashiwaya et al. ${ }^{18}$, who find a difference in CBM levels of $150 \mathrm{meV}$ to $450 \mathrm{meV}$. Their values for Fermi level position and VBM are based 
on photoemission experiments, but as photoemission accesses only occupied states, they had to derive their CBM from an otherwise determined bulk band gap. The Fermi level position in a DFT calculation of a semiconductor is, on the other hand, not very physical and placed on top of the highest occupied state here, so it cannot be compared to the experimental values. This shows that experimental band positions from photoelectron spectroscopy (PES) in vacuum with potentially only partial coverages are expected to deviate significantly from measurements of fully immersed surfaces or from ambient-pressure PES, where thin water layers are conserved. For molecular dynamics simulations, where timescales are often too short to account for surface chemical reactions, the large band position difference would, in combination with limited slab sizes, lead to large fluctuations in the band positions over time. This has indeed been observed by Guo et al. ${ }^{14}$, where they could not identify a single final model for anatase $\mathrm{TiO}_{2}$ from the $\mathrm{MD}$ simulations alone, albeit already for the (101) surface. Our results suggest that the situation for the (001) surface is even more challenging.

\section{CONCLUSION}

Our investigations into the (101) and (001) surfaces of anatase in contact with water reveal a rich surface chemistry of anatase, especially in the case of the (001) surface. For both surfaces, we identify considerable variations in the electronic structures and resulting band levels that depend on water coverage and degree of dissociation. These variations can both, improve and diminish the performance of anatase nanocrystals as photocatalysts, by modifying the relative band alignment as underlying driving force for electron-hole separation. Analysis of the densities of states for the different structures allowed us to identify an occupied, Ti-related surface state for the (001) surface. Its passivation by dissociative adsorption of water plays a crucial role in the observed band level variations. Our results suggest that, especially for the (001) surface, a wide tuning of the band positions and hence charge-transfer properties for both, solid-solid and solid-electrolyte interfaces should be possible by specifically conserving or passivating surface states. Preferential formation of $\{001\}$ facets can be facilitated in the presence of fluorine ions. ${ }^{34}$ The combination with subsequent, selective underpotential deposition ${ }^{35}$ of different catalysts or not covalently bonded passivation layers such as graphene (oxide), could then, in principle, allow for the control of relative band positions. Alternatively, physical methods such as a combination of annealing and oxidation steps can be used to fine-tune the density of surface states ${ }^{18}$ and, consequently, 
also band positions. We expect such modifications to allow for considerable improvements on the performance of anatase-based photocatalysts.

\section{SUPPLEMENTARY MATERIAL}

See supplementary material for different views of the relaxed structures as well as local density of states for the topmost layer of the bare (001) surface.

\section{ACKNOWLEDGMENTS}

Computational resources were provided UK Car-Parrinello (UKCP) consortium, funded by the Engineering and Physical Sciences Research Council (EPSRC) of the United Kingdom. The authors thank W. Jaegermann and B. Kaiser for edifying discussions. MMM acknowledges funding from the fellowship programme of the German National Academy of Sciences Leopoldina, grant LPDS 2015-09, and the German Research Foundation, project 434023472. Part of the work was funded by the Volkswagen Foundation and the International Exchanges grant IE161814 of the Royal Society.

This article has been accepted by the Journal of Chemical Physics. After it is published, it will be found at https://doi.org/10.1063/5.0004779.

\section{DATA AVAILABILITY STATEMENT}

The data that supports the findings of this study are available from the corresponding author upon reasonable request.

\section{REFERENCES}

${ }^{1}$ A. Fujishima and K. Honda, Nature 238, 37 (1972).

${ }^{2}$ B. Neumann, P. Bogdanoff, and H. Tributsch, Journal of Physical Chemistry C 113, 20980 (2009).

${ }^{3}$ C. N. Sayers and N. R. Armstrong, Surface Science 77, 301 (1978).

${ }^{4}$ J. P. W. Treacy, H. Hussain, X. Torrelles, D. C. Grinter, G. Cabailh, O. Bikondoa, C. Nicklin, S. Selçuk, A. Selloni, R. Lindsay, and G. Thornton, Physical Review B 95, 075416 (2017). 
${ }^{5}$ C. Dette, M. A. Pérez-Osorio, S. Mangel, F. Giustino, S. J. Jung, and K. Kern, Journal of Physical Chemistry C 122, 11954 (2018).

${ }^{6}$ P. J. D. Lindan, J. Muscat, S. Bates, N. M. Harrison, and M. Gillan, Faraday Discussions 106, 135 (1997).

${ }^{7}$ S. Selçuk and A. Selloni, The Journal of Physical Chemistry C 117, 6358 (2013).

${ }^{8}$ J. Cheng, X. Liu, J. A. Kattirtzi, J. VandeVondele, and M. Sprik, Angewandte Chemie International Edition 53, 12046 (2014).

${ }^{9}$ J. Yu, J. Low, W. Xiao, P. Zhou, and M. Jaroniec, Journal of the American Chemical Society 136, 8839 (2014), https://doi.org/10.1021/ja5044787.

${ }^{10}$ Y. Li and Y. Gao, Physical Review Letters 112, 206101 (2014).

${ }^{11}$ C. E. Patrick and F. Giustino, Physical Review Applied 2, 014001 (2014).

${ }^{12}$ S. Selçuk and A. Selloni, Journal of Physics D: Applied Physics 50, 273002 (2017).

${ }^{13}$ S. Selçuk and A. Selloni, Nature Materials 15, 1107 (2016).

${ }^{14}$ Z. Guo, F. Ambrosio, W. Chen, P. Gono, and A. Pasquarello, Chemistry of Materials 30, 94 (2018), https://doi.org/10.1021/acs.chemmater.7b02619.

${ }^{15}$ C. Zhang, J. Hutter, and M. Sprik, Journal of Physical Chemistry Letters 10, 3871 (2019).

${ }^{16}$ Y. He, A. Tilocca, O. Dulub, A. Selloni, and U. Diebold, Nature Materials 8, 585 (2009).

${ }^{17}$ M. Xu, Y. Gao, E. M. Moreno, M. Kunst, M. Muhler, Y. Wang, H. Idriss, and C. Wöll, Physical Review Letters 106, 138302 (2011).

${ }^{18}$ S. Kashiwaya, T. Toupance, A. Klein, and W. Jaegermann, Advanced Energy Materials 8, 1802195 (2018), https://onlinelibrary.wiley.com/doi/pdf/10.1002/aenm.201802195.

${ }^{19}$ S. Kashiwaya, J. Morasch, V. Streibel, T. Toupance, W. Jaegermann, and A. Klein, Surfaces 1, 73 (2018).

${ }^{20}$ I. Beinik, A. Bruix, Z. Li, K. C. Adamsen, S. Koust, B. Hammer, S. Wendt, and J. V. Lauritsen, Physical Review Letters 121, 206003 (2018).

${ }^{21}$ F. De Angelis, C. Di Valentin, S. Fantacci, A. Vittadini, and A. Selloni, Chemical Reviews 114, 9708 (2014).

${ }^{22}$ X.-Q. Gong and A. Selloni, Journal of Physical Chemistry B 109, 19560 (2005).

${ }^{23}$ U. Diebold, Surface Science Reports 48, 53 (2003).

${ }^{24}$ J. Hutter, M. Iannuzzi, F. Schiffmann, and J. VandeVondele, Wiley Interdisciplinary Reviews: Computational Molecular Science 4, 15 (2014).

${ }^{25}$ J. VandeVondele and J. Hutter, Journal of Chemical Physics 127, 114105 (2007). 
Band Positions of Anatase by DFT

${ }^{26}$ C. Hartwigsen, S. Goedecker, and J. Hutter, Physical Review B 58, 3641 (1998).

${ }^{27}$ J. P. Perdew, K. Burke, and M. Ernzerhof, Physical Review Letters 77, 3865 (1996).

${ }^{28}$ J. Heyd, G. E. Scuseria, and M. Ernzerhof, The Journal of Chemical Physics 118, 8207 (2003).

${ }^{29}$ M. Guidon, J. Hutter, and J. VandeVondele, Journal of Chemical Theory and Computation 6, 2348 (2010).

${ }^{30}$ A. Selloni, Nature Materials 7, 613 (2008).

${ }^{31}$ A. Vittadini, A. Selloni, F. P. Rotzinger, and M. Grätzel, Physical Review Letters 81, 2954 (1998).

${ }^{32}$ A. J. Samin and C. D. Taylor, Applied Surface Science 423, 1035 (2017).

${ }^{33}$ T. Le Bahers, M. Rérat, and P. Sautet, J. Phys. Chem. C 118, 5997 (2014).

${ }^{34}$ H. G. Yang, C. H. Sun, S. Z. Qiao, J. Zou, G. Liu, S. C. Smith, H. M. Cheng, and G. Q. Lu, Nature 453, 638 (2008).

${ }^{35}$ D. Liang and G. Zangari, Langmuir 30, 2566 (2014). 


\section{Supplementary Material - Band Positions of Anatase (001) and (101) Surfaces in Contact with Water from Density Functional Theory \\ Julian Geiger, ${ }^{1,2}$ Michiel Sprik, ${ }^{1,}$ a) and Matthias M. May ${ }^{1,3}$ \\ 1) Department of Chemistry, University of Cambridge, United Kingdom \\ 2) Friedrich-Alexander University Erlangen-Nürnberg, Germany ${ }^{\text {b) }}$ \\ ${ }^{3)}$ Institute for Solar Fuels, Helmholtz-Zentrum Berlin für Materialien und Energie GmbH, Germany $^{\mathrm{c})}$}

\footnotetext{
a)Electronic mail: ms284@cam.ac.uk

b) Present address: Institut Català d'Investigació Química, Tarragona, Spain

${ }^{c}$ Present address: Institute of Theoretical Chemistry, Ulm University, Germany
} 


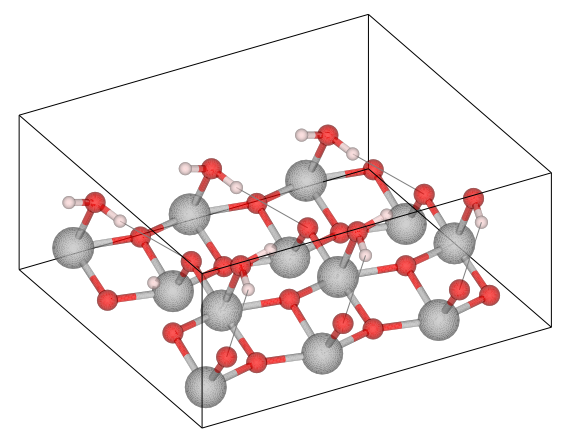

(a) $1 \mathrm{ML}$

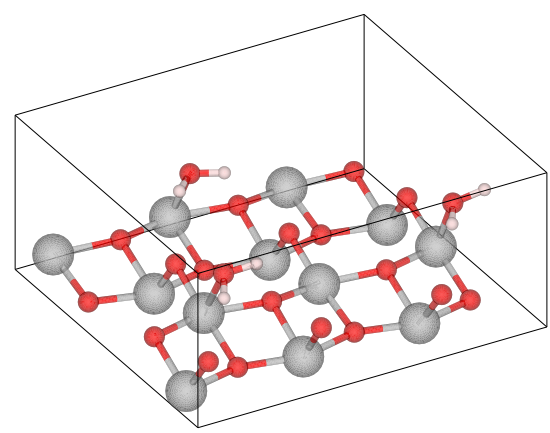

(b) $1 / 2 \mathrm{ML}$

FIG. S1. Spatial view of water adsorbed on the (101) surface, with one (a) and half a monolayer (b) coverage, corresponding to the top views in Fig. 2 in the main manuscript. Hydrogen bonds are represented as thin grey lines.

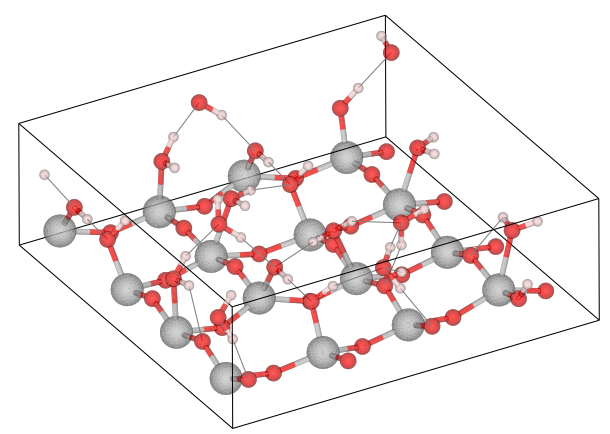

(a) $1 \mathrm{ML}$

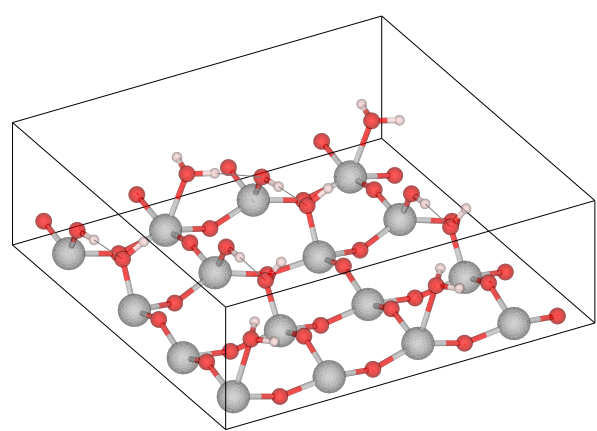

(b) $1 / 2 \mathrm{ML}$

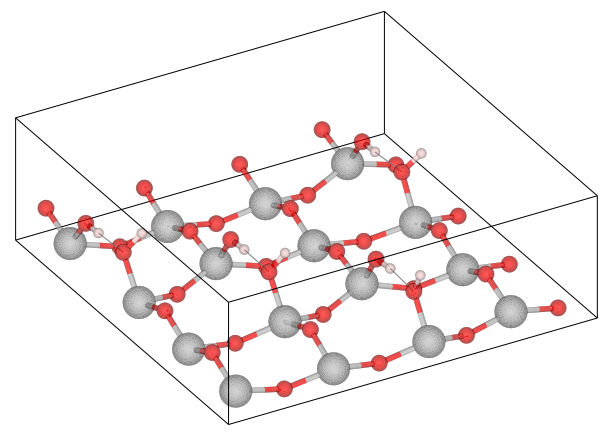

(c) $1 / 4 \mathrm{ML}$

FIG. S2. Spatial view of mixed (a,b) and fully dissociative (c) water adsorption on the (001) surface from different water coverages, corresponding to the top views in Fig. 3 in the main manuscript. 
Band Positions of Anatase by DFT

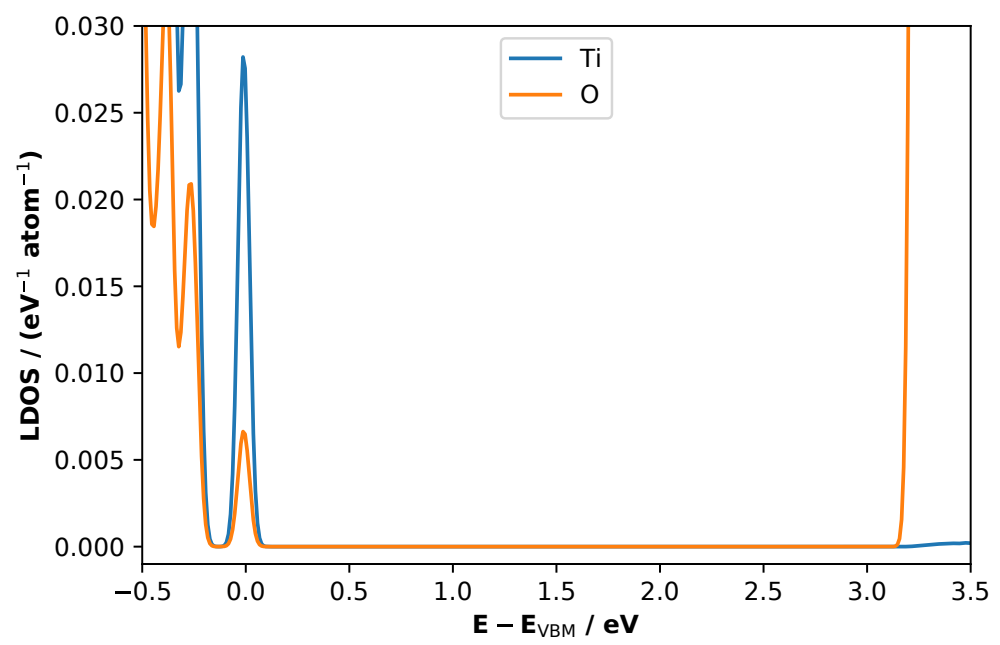

FIG. S3. Partial density of states for topmost Ti-O layer of the pristine (001) surface. The occupied surface state is clearly visible, the Fermi level is set to the highest occupied state. 
